\title{
La pluripatologia en una Unidad de Insuficiencia Cardíaca: la perspectiva de un internista
}

\author{
Pluripathology in a Heart Failure Unit - a internist' perspective
}

\author{
Marta Rafael Marques ${ }^{1}$, Helena Gonçalves ${ }^{2}$, Joana Calvao², Josebe Gairigolzarri ${ }^{3}$, \\ Manuel Mendez ${ }^{3}$, Ramon Bover ${ }^{3}$, Isidre Vilacosta ${ }^{3}$, Carlos Macaya ${ }^{3}$ \\ ${ }^{1}$ Centro Hospitalar Povoa de Varzim/Vila do Conde. Povoa de Varzim, Portugal. ${ }^{2}$ Centro Hospitalar de Trás-os-Montes e Alto Douro. Vila Real, Portugal. \\ ${ }^{3}$ Hospital Clínico San Carlos. Madrid, España
}

\section{RESUMEN}

Objetivo: Determinar la frecuencia de pluripatología en una Unidad de Insuficiencia Cardíaca, definir las características de los pacientes pluripatológicos y su pronóstico vital según dos modelos: el índice PROFUND y el Seattle Heart Failure Model.

Material y métodos: Se han analizado de forma consecutiva los pacientes observados en la consulta de insuficiencia cardíaca de un hospital de tercer nivel durante dos meses. Se han registrado comorbilidades, categorías de pluripatología y los índices de Charlson, Barthel, Seattle Heart Failure Model y PROFUND.

Resultados: Se incluyeron 246 pacientes, de los que 118 (48\%) fueron pluripatológicos, con índice de Charlson 7,9 $9 \pm 3,8$ y PROFUND 3,5 $\pm 7,1$. La categoría de pluripatología más prevalente fue la $A$, seguida de la $B$ y C. Los pluripatológicos fueron mayores (77 vs. 73 años, $p=0,001$ ), más frágiles, con mayor limitación funcional (Barthel: 84,7 vs. 96,1, p<0,001), mayor prevalencia de factores de riesgo cardiovascular y enfermedades crónicas e ingresaron más (14,4 vs. 4,7, p=0,015). La etiología más frecuente fue la cardiopatía isquémica. Los pacientes pluripatológicos tenían clase funcional más avanzada NHYA III-IV (4,2 vs. 0,8, $p<0,001)$, NT-proBNP más elevados $(2985 \mathrm{pg} / \mathrm{ml}$ vs. $1780 \mathrm{pg} / \mathrm{ml}, \mathrm{p}=0,013)$ y precisaron mayor dosis de diuréticos (60 vs $40, p<0,001$ ). Se verificó una concordancia en la estimativa de mortalidad entre el PROFUND y el Seattle Heart Failure Model.

Conclusiones: Este estudio demuestra la elevada frecuencia de pacientes pluripatológicos en una unidad de insuficiencia cardíaca, reflejando una mayor sobrecarga asistencial y necesidad de cuidados más complejos. Se trata de una población con gran fragilidad, dependencia funcional y comorbilidad, que obliga a plantear un abordaje multidisciplinar.

Palabras clave: Insuficiencia cardíaca; Pluripatología; Pronóstico; Mortalidad; PROFUND.

\section{INTRODUCCIÓN}

Los cambios sociosanitarios y los avances en técnicas diagnósticas y terapéuticas han permitido aumentar la esperanza de vida de forma significativa en los últimos años. Esto se ha asociado a un aumento en la incidencia y prevalencia de patologías crónicas, como la Insuficiencia Cardiaca (IC), entidad cada vez más frecuente y que supone un problema sociosanitario de primer orden ${ }^{1}$. Asociado a ello, los pacientes de estas dolencias son cada vez más pluripatológicos (PP), definido como la coexistencia de dos o más enfermedades crónicas que conlleva la aparición de patologías interrelacionadas ${ }^{2}$.

\begin{abstract}
Objectives: to determine the frequency of pluripathology in a Heart Failure Unit, defining the characteristics of pluripathological patients and their vital prognosis according to two models: PROFUND score and Seattle Heart Failure Model.

Methods: consecutive patients from a Heart Failure Unit of a third level hospital were analized during two months. Comorbidities, pluripathology categories and Charlson, Barthel, Seattle Heart Failure Model and PROFUND scores were registered.

Results: 246 patients were included, of which $118(48 \%)$ were pluripathological, with Charlson score $7.9 \pm 3.8$ and PROFUND $3.5 \pm 7.1$. The most prevalent category of pluripathology was $A$, followed by $B$ and C. The pluripathological patients were older (77 vs. 73 years, $p=0.001$ ), more fragile, with greater functional limitation (Barthel: 84.7 vs. 96, 1 , $p<0.001$ ), higher prevalence of cardiovascular risk factors and chronic diseases and admitted more (14.4 vs. 4.7, $p=0.015$ ). The most frequent etiology was ischemic heart disease. The pluripathological patients had more advanced functional class NHYA III-IV (4.2 vs. 0.8, $p<0.001$ ), higher NT-proBNP (2985 pg/ml vs. $1780 \mathrm{pg} / \mathrm{ml}, \mathrm{p}=0.013)$ and required higher dose of diuretics ( 60 vs $40, p<0.001$ ). A concordance in the mortality estimate between the PROFUND and the Seattle Heart Failure Model was verified.

Conclusion: Our study demonstrates the high frequency of pluripathological patients in a heart failure unit, population with great fragility, due to functional dependence and the association of comorbidities, that requires a multidisciplinary approach.
\end{abstract}

Keywords: Hearth feilure; Pluripathology; Prognosis; Mortality; PROFUND.

Los PP constituyen un grupo de gran complejidad y vulnerabilidad clínica, con elevada dependencia funcional y frecuente deterioro cognitivo asociado. En general presentan especial fragilidad clínica, que constituye un factor de riesgo para el desarrollo de múltiples eventos adversos y que conduce frecuentemente a una mayor dependencia. Todo ello produce una elevada morbimortalidad durante los ingresos de estos pacientes, así como un gasto sociosanitario muy elevado ${ }^{3-6}$. Se estima que, en el entorno hospitalario, la prevalencia de PP es del 25-50\% según las áreas y la tasa de mortalidad alcanza el 37-38\% al año. Esta mortalidad es 
significativamente superior a la de los pacientes no pluripatológicos ${ }^{7-9}$. Cabe destacar que la presencia de pacientes PP es extensible a la mayoría de las especialidades clínicas, habiéndose descrito una prevalencia de pacientes de este tipo de hasta el 36,9\% en una unidad de hospitalización de Cardiología, provocando una mayor sobrecarga asistencial y cuidados multidisciplinares ${ }^{10}$.

Pese a la alta prevalencia, los pacientes con comorbilidades y pluripatología siguen excluidos de la mayoría de los ensayos clínicos y estudios de intervención o registros. Sin embargo, es de vital importancia establecer un pronóstico vital y funcional de estos pacientes en la práctica médica diaria, para una correcta planificación de las metas y objetivos diagnósticos y terapéuticos. Estas decisiones son útiles tanto para intensificar las estrategias del control del riesgo cardiovascular y enfermedades crónicas asociadas como para seleccionar a los pacientes que se benefician de atención paliativa, evitando la iatrogenia y la futilidad ${ }^{11}$.

De entre las herramientas de evaluación pronóstica, el clásico índice de comorbilidad de Charlson es el más utilizado y relaciona el peso de la comorbilidad con la supervivencia a los 10 años ${ }^{12}$. La mayoría de los índices pronósticos son desarrollados para poblaciones concretas, como es el Seattle Heart Failure Model (SHFM) para estimar la supervivencia en pacientes con insuficiencia cardíaca ${ }^{13}$.

Más recientemente se ha desarrollado el índice PROFUND (PROpredicción FUNcional Desarrollado para pacientes pluripatológicos), especialmente diseñado para pacientes pluripatológicos. Este índice fue elaborado en un estudio multicéntrico de hospitales españoles por un grupo de trabajo sobre pacientes pluripatológicos, y posteriormente validado externamente ${ }^{8,9}$. Se basa en 9 variables demográficas, clínicas, analíticas y sociofamiliares y clasifica a los pacientes en 4 grupos de riesgo de mortalidad, desde riesgo bajo (12$14 \%$ ) hasta riesgo muy alto (61-68\%). El índice PROFUND predice la mortalidad al cabo de un año en este tipo de pacientes y también ha demostrado su buena capacidad pronóstica en el seguimiento a 4 años ${ }^{14}$.

El objetivo de este estudio fue determinar la frecuencia de pluripatología en una unidad de IC de Cardiología, definir las características basales de los PP y su pronóstico. Además, se pretende comparar los resultados obtenidos al aplicar el SHFM con el índice PROFUND.

\section{MATERIAL Y MÉTODOS}

Estudio prospectivo observacional, en el que se incluyeron consecutivamente todos los pacientes atendidos en la consulta de una Unidad de Insuficiencia Cardíaca (UIC) de Cardiología de un hospital de tercer nivel por un período de dos meses. Se excluyeron las reevaluaciones de pacientes previamente incluidos en este periodo.

La recogida de datos se realizó de forma directa mediante entrevista presencial estructurada con el paciente y consulta de la historia clínica electrónica. Se recogieron las principa- les características demográficas, clínicas y analíticas de los pacientes. Se determinó la comorbilidad según el índice de Charlson ${ }^{12}$, la dependencia según el índice de Barthel15 y la fragilidad según el Clinical Frailty Scale ${ }^{16}$. Se recogieron el número de ingresos en el último año por descompensación de la IC, categorías y criterios de pluripatologia².

Aquellos pacientes que cumplieron criterios de PP se les calculó el índice PROFUND ${ }^{8}$. El índice PROFUND se calculó según sus 9 variables que determinan su puntuación: edad $\geq 85$ años (3 puntos), cuidador principal distinto al cónyuge (2 puntos), neoplasia activa (6 puntos), demencia (3 puntos), disnea clase III-IV de la NYHA o la escala Medical Research Council (3 puntos), delirium en el último ingreso hospitalario (3 puntos), hemoglobina $<10 \mathrm{~g} / \mathrm{dl}$ (3 puntos), índice de Barthel $<60$ (4 puntos), $\geq 4$ ingresos hospitalarios en los 12 meses previos (3 puntos). Esto permite clasificar a los pacientes en 4 grupos de riesgo de muerte al año: riesgo bajo (0-2 puntos; 12.1-14.6\%), riesgo medio (3-6 puntos; $21.5-31.5 \%)$, riesgo alto (7-10 puntos; $45-50 \%$ ) y riesgo muy alto ( $\geq 11$ puntos; $61.3-68 \%$ ). Por su vez, a todos los pacientes se les aplicó el índice de predicción de mortalidad SHFM según sus 20 variables, que requiere la utilización de una calculadora disponible en la web (http://www. SeattleHeartFailureModel.org). Además, se compararon los resultados de predicción de mortalidad del SHFM según la escala de PROFUND.

Análisis estadístico: Las variables cualitativas se expresaron en porcentajes y se analizaron los contrastes de asociación mediante el test de Chi cuadrado o, en su defecto, el test de Fischer. Las variables cuantitativas se expresaron como media (desviación estándar), su distribución fue analizada con el test de Kolmogorov-Smirnov, y las diferencias se analizaron mediante la $t$ de Student para las variables que seguían una distribución normal y con la prueba U de MannWhitney en caso contrario. El análisis de supervivencia se realizó con el método de Kaplan-Meier y su análisis multivariado mediante regresión de COX. Todos los resultados fueron analizados mediante el programa estadístico SPSS (Statistical Package for the SocialSciences, versión 20.0; IBM Corp.; Armonk, Nueva York, Estados Unidos). El nivel de significación estadística se estableció para un valor de $p<$ 0,05 . El diseño y la metodología del estudio se realizaron cumpliendo con la Declaración de Helsinki de 1975 y fue aprobado por el CEIC Hospital Clínico San Carlos.

\section{RESULTADOS}

Se incluyeron un total de 246 pacientes, con una edad media de $75 \pm 11,9$ años, de los que 104 (42,3\%) fueron mujeres. Las características descriptivas de los pacientes se presentan en la tabla 1.118 (48\%) pacientes fueron pluripatológicos. Los PP eran de mayor edad $(77 \pm 10,1$ vs $73 \pm 13,1$; $p=0,010)$ y tenían más dependencia para las actividades básicas e instrumentales de la vida diaria (Índice de Barthel: $84,7 \pm 18,4$ vs $96,1 \pm 7,1 ; p<0,001)$. Además, estos pacientes eran más frágiles (Clinical Frailty Scale: moderadamente 
Tabla 1. Características clínicas de la muestra y análisis del subgrupo de pacientes pluripatológicos.

\begin{tabular}{|c|c|c|c|c|}
\hline Características clínicas & Muestra & No PP & PP & Valor de $p$ \\
\hline Total pacientes & $246(100)$ & $128(52)$ & $118(48)$ & \\
\hline \multicolumn{5}{|l|}{ Sexo } \\
\hline Femenino & $104(42,3)$ & $62(48,4)$ & $42(35,6)$ & \multirow{2}{*}{$p=0,042$} \\
\hline Masculino & $142(57,7)$ & $66(51,6)$ & $76(64,4)$ & \\
\hline Edad (años) ${ }^{\mathrm{a}}$ & $75 \pm 11,9$ & $73 \pm 13,1$ & $77 \pm 10,1$ & $p=0,010$ \\
\hline Índice de Charlson ${ }^{\mathrm{a}}$ & $6,4 \pm 3,4$ & $4,9 \pm 2,1$ & $7,9 \pm 3,8$ & $p<0,001$ \\
\hline Índice de Barthel ${ }^{\mathrm{a}}$ & $90,6 \pm 14,8$ & $96,1 \pm 7,1$ & $84,7 \pm 18,4$ & $p<0,001$ \\
\hline \multicolumn{5}{|l|}{ Clinical Frailty Scale $^{b}$} \\
\hline Atlético & $35(17,8)$ & $29(29,6)$ & $6(6,1)$ & \multirow{7}{*}{$p<0,001$} \\
\hline Buen estado físico & $43(21,8)$ & $24(24,5)$ & $19(19,2)$ & \\
\hline $\begin{array}{l}\text { Buen estado físico con } \\
\text { enfermedad estable }\end{array}$ & $44(22,3)$ & $24(24,5)$ & $20(20,2)$ & \\
\hline Aparentemente vulnerable & $30(15,2)$ & $12(12,3)$ & $18(18,2)$ & \\
\hline Levemente frágil & $14(7,1)$ & $7(7,1)$ & $7(7,1)$ & \\
\hline Moderadamente frágil & $17(8,7)$ & $2(2,0)$ & $15(15,1)$ & \\
\hline Gravemente frágil & $14(7,1)$ & - & $14(14,1)$ & \\
\hline \multicolumn{5}{|l|}{ Factores de riesgo cardiovascular } \\
\hline Hipertensión arterial & $181(73,6)$ & $85(66,4)$ & $96(81,4)$ & $p=0,008$ \\
\hline Diabetes mellitus & $98(39,8)$ & $31(24,2)$ & $67(56,8)$ & $p<0,001$ \\
\hline Dislipidemia & $152(61,8)$ & $66(51,6)$ & $86(72,9)$ & $p=0,010$ \\
\hline \multicolumn{5}{|l|}{ Tabaquismo } \\
\hline Fumador activo & $37(15)$ & $19(14,8)$ & $18(15,3)$ & \multirow{2}{*}{$p=0,420$} \\
\hline Exfumador & $56(22,8)$ & $25(19,5)$ & $31(26,3)$ & \\
\hline Obesidad & $66(26,8)$ & $32(25,2)$ & $34(29,1)$ & $p=0,497$ \\
\hline IMC $\left(\mathrm{kg} / \mathrm{m}^{2}\right)^{\mathrm{a}}$ & $27 \pm 4,8$ & $26,3 \pm 4,4$ & $27,8 \pm 5$ & $p=0,016$ \\
\hline \multicolumn{5}{|l|}{ Otras enfermedades } \\
\hline Fibrilación auricular & $149(60,6)$ & $66(51,6)$ & $83(70,3)$ & $p=0,003$ \\
\hline Enfermedad coronaria & $92(37,4)$ & $35(27,3)$ & $57(48,3)$ & $p=0,001$ \\
\hline Enfermedad arterial periférica & $44(17,9)$ & $16(12,5)$ & $28(23,7)$ & $p=0,022$ \\
\hline Enfermedad cerebrovascular & $31(12,6)$ & $11(8,6)$ & $20(16,9)$ & $p=0,049$ \\
\hline Enfermedad renal crónica & $137(55,7)$ & $48(38,7)$ & $89(75,4)$ & $p<0,001$ \\
\hline $\mathrm{FG}\left(\mathrm{ml} / \mathrm{min} / 1,73 \mathrm{~m}^{2}\right)^{\mathrm{a}}$ & $58,7 \pm 22,7$ & $67,7 \pm 21,3$ & $49,5 \pm 20,4$ & $p<0,001$ \\
\hline Anemia & $62(25,2)$ & $25(20,5)$ & $37(31,4)$ & $p=0,055$ \\
\hline Enfermedad Pulmonar Obstructiva Crónica & $41(16,7)$ & $10(7,8)$ & $31(26,3)$ & $p<0,001$ \\
\hline Síndrome Apena Obstructiva del Sueño & $32(13)$ & $10(7,8)$ & $22(18,6)$ & $p=0,012$ \\
\hline Neoplasias & $47(19,1)$ & $20(15,6)$ & $27(22,9)$ & $p=0,148$ \\
\hline Trastorno por ansiedad o depresión & $47(19,1)$ & $19(14,8)$ & $28(23,7)$ & $p=0,077$ \\
\hline Patología de tiroides & $33(13,4)$ & $14(10,9)$ & $19(16,1)$ & $p=0,235$ \\
\hline \multicolumn{5}{|l|}{ Datos analíticos } \\
\hline Hemoglobina $(\mathrm{g} / \mathrm{dL})^{\mathrm{a}}$ & $13,1 \pm 1,8$ & $13,5 \pm 1,7$ & $12,9 \pm 1,9$ & $p=0,012$ \\
\hline Linfocitos (\%) ${ }^{\mathrm{a}}$ & $24,4 \pm 10,2$ & $24,6 \pm 9,8$ & $24,3 \pm 19,6$ & $p=0,798$ \\
\hline Creatinina plasmática (mg/dL) ${ }^{\mathrm{a}}$ & $1,2 \pm 0,6$ & $1,0 \pm 0,3$ & $1,5 \pm 0,7$ & $p<0,001$ \\
\hline Colesterol total $(\mathrm{mg} / \mathrm{dl})^{\mathrm{a}}$ & $152,6 \pm 39,6$ & $164,9 \pm 39,9$ & $140,2 \pm 35,3$ & $p<0,001$ \\
\hline Colesterol LDL (mg/dl) ${ }^{\mathrm{a}}$ & $81,6 \pm 30,8$ & $90,8 \pm 31,4$ & $72,4 \pm 27,4$ & $p<0,001$ \\
\hline NT-proBNP (pg/mL), P50 & & 1780,0 & 2985,0 & $p=0,013$ \\
\hline
\end{tabular}

frágil 15 vs 2; gravemente frágil 14 vs $0 ; p<0,001$ ) y pre-

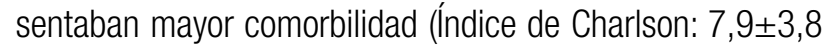
vs $4,9 \pm 2,1 ; p<0,001)$. Los PP presentaron mayor prevalencia de factores de riesgo cardiovascular y se verificó mayor prevalencia de enfermedades cardiovasculares, nefrológicas y respiratorias. Los PP tenían niveles más bajos de colesterol total $(140,2 \pm 35,3$ vs $164,9 \pm 39,9 ; p<0,001)$ y valores más elevados de NT-proBNP (2985,0 vs 1780,0; $p=0,013)$.
IMC - índice masa corporal; FG - filtrado glomerular. Los datos se expresan en valor absoluto (porcentaje) y ${ }^{a}$ media \pm desviación estándar. ${ }^{\mathrm{b}}$ Datos de 197 pacientes (Clinical Frailty Scale se evalúa en pacientes con edad $>65$ años, excluidos 49 pacientes).

La tabla 2 recoge las características diferenciales entre los PP y no pluripatológicos en relación a insuficiencia cardíaca. Los PP presentaron clase funcional más avanzada de la 
Tabla 2. Insuficiencia cardíaca y análisis del subgrupo de pacientes pluripatológicos.

\begin{tabular}{|c|c|c|c|c|c|}
\hline Insuficiencia Cardíaca & Muestra & \multicolumn{2}{|c|}{ No PP } & PP & Valor de $p$ \\
\hline \multicolumn{6}{|l|}{ Clase funcional - NHYA } \\
\hline I & $44(17,9)$ & \multicolumn{2}{|c|}{$33(25,8)$} & $11(9,3)$ & \multirow{4}{*}{$p<0,001$} \\
\hline$\|$ & $139(56,5)$ & \multicolumn{2}{|c|}{$74(57,8)$} & $65(55,1)$ & \\
\hline III & $57(23,2)$ & \multicolumn{2}{|c|}{$20(15,6)$} & $37(31,4)$ & \\
\hline IV & $6(2,4)$ & \multicolumn{2}{|c|}{$1(0,8)$} & $5(4,2)$ & \\
\hline \multicolumn{6}{|l|}{ DSVE } \\
\hline Deprimida & $74(30,1)$ & \multicolumn{2}{|c|}{$36(28,3)$} & $38(32,2)$ & \multirow{3}{*}{$p=0,68 c$} \\
\hline Intermedia & $64(26,0)$ & \multicolumn{2}{|c|}{$33(26)$} & $31(26,7)$ & \\
\hline Preservada & $105(42,7)$ & \multicolumn{2}{|c|}{$58(45,7)$} & $47(40,5)$ & \\
\hline $\mathrm{FEVl}^{\mathrm{a}}$ & & \multicolumn{2}{|c|}{$47,5 \pm 14,1$} & $45,7 \pm 13,6$ & $p=0,310$ \\
\hline \multicolumn{6}{|l|}{ Etiología } \\
\hline Cardiopatía isquémica & $94(38,2)$ & \multicolumn{2}{|c|}{$38(29,7)$} & $56(47,5)$ & \\
\hline Cardiopatía valvular & $56(22,8)$ & \multicolumn{2}{|c|}{$30(23,4)$} & $26(22)$ & \\
\hline Taquimiocardiopatia & $31(12,6)$ & \multicolumn{2}{|c|}{$17(13,3)$} & $14(11,9)$ & \\
\hline Cardiopatía hipertensiva & $11(4,5)$ & \multicolumn{2}{|c|}{$4(3,1)$} & $7(5,9)$ & \\
\hline Alcohólica & $5(2,0)$ & \multicolumn{2}{|c|}{$3(2,3)$} & $2(1,7)$ & \\
\hline Quimioterapia & $9(3,7)$ & \multicolumn{2}{|c|}{$6(4,7)$} & $3(2,5)$ & \\
\hline Hipertrófica no obstructiva & $5(2,0)$ & \multicolumn{2}{|c|}{$5(3,9)$} & - & \\
\hline Miocardiopatía no compactada & $2(0,8)$ & \multicolumn{2}{|c|}{$2(1,6)$} & - & \\
\hline Idiopática & $23(9,3)$ & \multicolumn{2}{|c|}{$18(14,1)$} & $5(4,2)$ & \\
\hline Investigación no concluida & $10(4,1)$ & \multicolumn{2}{|c|}{$5(3,9)$} & $5(4,2)$ & \\
\hline \multicolumn{6}{|c|}{ Ingresos en últimos 12 meses por IC } \\
\hline 0 & $168(68,3)$ & $96(75,0)$ & $72(61,0)$ & \multirow{3}{*}{\multicolumn{2}{|c|}{$p=0,015$}} \\
\hline 1 & $55(22,4)$ & $26(20,3)$ & $29(24,6)$ & & \\
\hline$\geq 2$ & $23(9,3)$ & $6(4,7)$ & $17(14,4)$ & & \\
\hline \multicolumn{6}{|l|}{ Medicación crónica } \\
\hline Betabloqueantes & $186(75,6)$ & \multicolumn{2}{|c|}{$99(77,3)$} & $87(74,4)$ & $p=0,585$ \\
\hline IECA/ARA-II & $147(59,8)$ & \multicolumn{2}{|c|}{$80(62,5)$} & $67(57,3)$ & $p=0,403$ \\
\hline Antagonistas de Aldosterona & $119(48,4)$ & \multicolumn{2}{|c|}{$57(44,5)$} & $62(53,0)$ & $p=0,186$ \\
\hline LCZ696 (Sacubitril/Nalsartan) & $45(18,3)$ & $22(17$ & & $23(19,7)$ & $p=0,618$ \\
\hline Diurético, P50 & & 40 & & 60,0 & $p<0,001$ \\
\hline Estatina & $143(58,1)$ & $63(45$ & & $80(68,4)$ & $p=0,002$ \\
\hline
\end{tabular}

NYHA - New York Heart Association; DSVI - Disfunción sistólica del ventrículo izquierdo; FEVI - Fracción de eyección del ventrículo izquierdo; IC - Insuficiencia cardíaca; IECA - Inhibidores de la Enzima convertidora de Angiotensina; ARA - Antagonistas del receptor de la angiotensina. Los datos se expresan en valor absoluto (porcentaje) y ${ }^{\text {a }}$ media \pm desviación estándar.

NYHA (III: 31,4 vs 15,6 ; IV: 4,2 vs 0,$8 ; p<0,001$ ), aunque no se evidenció diferencia estadísticamente significativa en la FEVI 0 en la etiología de la cardiopatía. Además, los PP ingresaron más en el último año por IC descompensada frente a los no pluripatológicos ( 14,4 vs 4,7; $p=0,015)$. No se verificaron diferencias estadísticamente significativas en los fármacos modificadores de pronóstico entre los grupos. Las estatinas fueron más utilizadas por los PP que, a su vez, presentaron valores más controlados de colesterol total y LDL. Los PP necesitaron de dosis más elevadas de diurético.

La tabla 3 y la figura 1 muestran la relación entre los instrumentos de evaluación que se han utilizado para estimar la mortalidad. Los PP tenían puntuaciones más altas en el SHFM (6,0 vs 4,0; $p<0,001)$. Del total de pacientes, 118 cumplían criterios de pluripatología y $44(37,3 \%)$ pacien- tes presentaban tres o más categorías. Todos los pacientes cumplieron la categoría definitoria A (enfermedades cardiacas) ya que se trata de una población con IC, seguida de las enfermedades renales y vasculitis (categoría B) en 82 pacientes $(33,3 \%$ ) y de las enfermedades neurológicas (categoría C) en 31 pacientes (12,6\%). El índice PROFUND medio calculado fue de $3,5 \pm 7,1$. Al estratificar a los pacientes según los niveles de riesgo del índice PROFUND, 46 (39,0\%) pacientes tenían un riesgo bajo de mortalidad al año, 55 $(46,6 \%)$ un riesgo intermedio, $8(6,8 \%)$ un riesgo alto y 9 $(7,6 \%)$ un riesgo muy alto.

El diagrama de cajas de la figura 1 refleja la redistribución de la predicción de mortalidad del SHFM según la escala de PROFUND exclusivamente en los PP. Se verificó una concordancia en la estimación de mortalidad entre ambos índices. 
Tabla 3. ÍNDICES de los pacientes no pluripatológicos y pluripatológicos.

\begin{tabular}{|l|c|c|c|}
\hline Estratificación de Mortalidad & No PP & PP & Valor de $p$ \\
\hline Índice Seattle, $P_{50}$ & 4,0 & 6,0 & $p<0,001$ \\
\hline Índice PROFUND & \multicolumn{3}{|l|}{} \\
\hline Categorías definitorias & \multicolumn{3}{|l|}{} \\
\hline A (enfermedades cardíacas) & & $118(100)$ & \\
\hline B (enfermedades renales y vasculitis) & & $82(69,5)$ & \\
\hline C (enfermedades pulmonares) & & $31(26,3)$ & \\
\hline E (enfermedades neurológicas) & & $27(22,9)$ & \\
\hline F (diabetes con neuropatía y arteriopatía) & & $21(17,8)$ & \\
\hline G (anemia y neoplasia) & & $16(13,6)$ & \\
\hline H (enfermedad osteoarticular) & & $14(11,9)$ & \\
\hline D (enfermedades digestivas) & & $1(0,8)$ & \\
\hline Número de categorías por paciente ${ }^{\mathrm{a}}$ & & $2,6 \pm 1,0$ & \\
\hline Pacientes con $\geq 3$ categorías & & $44(37,3)$ & \\
\hline
\end{tabular}

Fig. 1. Diagrama de cajas que refleja la distribución del Seattle según la escala de PROFUND.

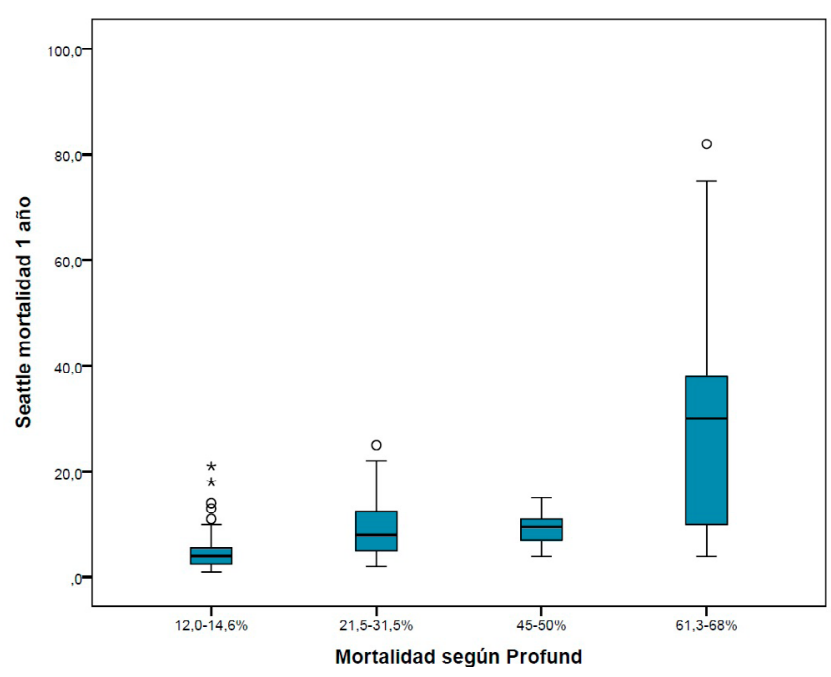

\section{DISCUSIÓN}

Este estudio demuestra la elevada frecuencia de pluripatología (48\% de la cohorte) en la práctica clínica diaria en pacientes evaluados en la consulta de una UIC de cardiología, superior incluso a lo publicado en planta de hospitalización de Cardiología (36,9\%). ${ }^{10}$ Paradójicamente, estos pacientes son frecuentemente excluidos de ensayos clínicos, ya que se tratan de pacientes mayores, con fragilidad, dependencia funcional y comorbilidad alta. Por ello, es de gran importancia evaluar a los pacientes de estas Unidades de forma multidimensional como el índice PROFUND. Además, el índice PROFUND ha permitido identificar que un porcentaje significativo de pacientes tiene una estimación de riesgo de mortalidad al año alto o muy alto, modificándose la estimación de mortalidad del SHFM para un determinado índice de PROFUND.
De acuerdo con otros estudios ${ }^{17}$, en pacientes con IC, se describe en la literatura una tasa de hospitalización por cualquier motivo al año de $70 \%$, aunque solo el $30 \%$ de los ingresos se deben a descompensación de la IC, con fuertes tendencias relacionadas con aumento de la edad y presencia de comorbilidad. Es probable que nuestra estimación de ingresos sea una subestimación, ya que solo se consideró ingresos donde la IC fue el diagnóstico principal.

Las comorbilidades no cardíacas son altamente prevalentes en pacientes con IC crónica y se asocian fuertemente con resultados clínicos adversos, como aumento del riesgo de hospitalización y muerte. En nuestro estudio, el peso de las comorbilidades fue elevado (6,4 puntos) e incluso mayor que el observado en el estudio SEMI-IC (5,4 puntos) $)^{18}$. Los factores de riesgo cardiovascular, la insuficiencia renal crónica y la enfermedad pulmonar son las comorbilidades más frecuentemente presentes a lo largo de la historia natural de la IC, lo que también se evidenció en nuestro conjunto de datos.

El uso del índice PROFUND ha permitido identificar que aproximadamente el $15 \%$ de los PP observados en la UIC presentan una alta 0 muy alta probabilidad de fallecer al cabo de un año y, casi la mitad presenta un riesgo intermedio (46,6\%). Además, estos pacientes presentaban peor clase funcional, mayor requerimiento diurético y habían presentado mayor número de ingresos en el año anterior. Sin embargo, no se objetivaron diferencias significativas en el manejo médico de la terapéutica modificadora de pronóstico de la IC entre los PP y no pluripatológicos. Estos datos confirman la gravedad de estos pacientes y sugieren que la alta carga de comorbilidades en su conjunto determina al menos en parte el pronóstico de los pacientes con IC, como se observa en la predicción de mortalidad del SHFM según el riesgo calculado por índice PROFUND.

Además, la polifarmacia en PP aumenta el riesgo de interacciones medicamentosas y efectos adversos que pueden llegar a ser más incapacitantes que la propia enfermedad cardíaca9 $^{9}$. El recurso de índices de pronóstico, como el 
SHFM específico de la IC, resulta de especial utilidad desde el punto de vista clínico y de planificación sanitaria. Sin embargo, según el informe de la OECD ${ }^{19}$, se calcula que solo el $3 \%$ de los pacientes con IC tienen la enfermedad de forma aislada, así que podría ser más apropiado utilizar índices específicamente desarrollados para pluripatología, como el índice PROFUND.

Así, pacientes de bajo-intermedio riesgo pueden beneficiarse de una intensificación de cuidados de salud, mientras que en los de alto riesgo de mortalidad se debería plantear el beneficio de intervenciones médicas exhaustivas y conducir a un inicio más temprano de tratamiento paliativo junto con el tratamiento cardiológico en pacientes con IC avanzada ${ }^{20}$. Todo esto justifica la necesidad de estratificación de los pacientes en función de su pronóstico para establecer objetivos terapéuticos que se ajusten a su situación personal. Un enfoque holístico integral de la IC y sus comorbilidades, en lugar de las estrategias tradicionales de manejo y del tratamiento individual de cada afección asociada, podría obtener mejores resultados en términos de pronóstico, mortalidad e ingresos. De hecho, los profesionales de salud que atienden habitualmente a PP con IC podrían mejorar los resultados en esta población de alto riesgo al identificar las condiciones no cardíacas y tratarlas desde el inicio del proceso asistencial.

\section{CONCLUSIONES}

Este estudio demuestra que la prevalencia de PP en una UIC de Cardiologia está en torno al 50\%, que refleja a su vez una mayor sobrecarga asistencial y necesidad de cuidados más complejos. Los PP definen un grupo de población con gran fragilidad, dependencia funcional y asociación de comorbilidades, que obliga a plantear un abordaje multidisciplinar con estrategias para el control del riesgo cardiovascular y de las enfermedades crónicas asociadas, así como adaptar los recursos sanitarios que más se ajusten a su expectativa de vida. El índice PROFUND parece una herramienta útil en el manejo clínico y su utilización podría ayudar en la planificación anticipada de decisiones.

\section{BIBLIOGRAFÍA}

1. Sayago-Silva I, García-López F, Segovia-Cubero J. Epidemiología de la insuficiencia cardiaca en España en los últimos 20 años. Rev Esp Cardiol. 2013:66(8)649-656

2. Ollero-Baturone M, Bernabeu-Wittel M, Espinosa Almendro J, García Estepa R, Morilla Herrera J, Pascual de la Pisa B, et al. Atención a Pacientes Pluripatológicos. Proceso Asistencial Integrado. $3^{\text {a }}$ edición. Consejería de Salud; 2018

3. Bernabeu-Wittel M, Jadad A, Moreno-Gaviño L, Hernández-Quiles C, Toscano F Cassani $\mathrm{M}$, et al. Peeking through the cracks: An assessment of the prevalence, clinical characteristics and health-related quality of life of people with polypathology in a hospital setting. Arch Gerontol Geriatr. 2010; 51:185-91.

4. Rincón-Gómez M, Bernabeu-Wittel M, Bohórquez-Colombo P, Moreno-Gaviño L, Cassani-Garza M, Ortiz-Camúñez MA, et al. Perceived quality of healthcare in a multicenter, community-based population of polypathological patients. Arch Gerontol Geriatr. 2011; 52:142-6.

5. Bernabeu-Wittel M, Barón-Franco B, Murcia-Zaragoza J, Fuertes-Martín A, RamosCantos C, Fernández-Moyano $A$, et al. on behalf of the PROFUND researchers. A multi-institutional, hospital-based assessment of clinical, functional, sociofamilial and health-care characteristics of polypathological patients. Arch Gerontol Geriatr. 2011; 53:284-91.

6. Ramírez-Duque N, Ollero-Baturone M, Bernabeu-Wittel M, Rincón-Gómez M, Ortiz-Camuñez MA, García-Morillo S. Características clínicas, funcionales, mentales y sociales de pacientes pluripatológicos. Estudio prospectivo durante un año en Atención Primaria. Rev Clin Esp. 2008; 208:4-11

7. Díez-Manglano J, Escalante Yangüela B, García-Arilla CalvoE, Ubis Díez E, Munilla López E, Clerencia Sierra M, et al. Differential characteristics in polypathological inpatients in internal medicine departments and acute geriatric units: The PLUPAR study. Eur J Intern Med. 2013; 24:767-71.

8. Bernabeu-Wittel M, Ollero-Baturone M, Moreno-Gaviño L, Barón-Franco B, Fuertes A, Murcia-Zaragoza J, et al. Development of a new predictive model for polypathological patients. The PROFUND index. Eur J Intern Med. 2011; 22:311-7

9. Díez-Manglano J, Cabrerizo García JL, García-Arilla Calvo E, et al. External validation of the PROFUND index in polypathological patients from internal medicine and acute geriatrics departments in Aragón. Intern Emerg Med. 2015; 10:915-26

10. López-Garrido MA, Martín-Portugués IA, Becerra-Muñoz VM, Orellana-Figueroa HN, Sánchez-Lora FJ, Morcillo-Hidalgo L et al. Prevalencia de pluripatología y valor pronóstico del índice PROFUND en una unidad de hospitalización de Cardiología. Rev Clin Esp. 2016;217(2):87-94

11. Bernabeu-Wittel M, Barón-Franco B, Nieto-Martín D, Moreno-Gaviño L, RamírezDuque N, Ollero-Baturone M. Estratificación pronóstica y abordaje asistencial de los pacientes pluripatológicos. Rev Clin Esp. 2017;217(7):410-419

12. Charlson ME, Pompei P, Ales KL, MacKenzie CR. A new method of classifying prognostic comorbidity in longitudinal studies: Development and validation. $\mathrm{J}$ Chronic Dis. 1987;40:373-83.

13. Levy WC, Mozaffarian D, Linker DT, Sutradhar SC, Anker SD, Cropp AB, et al. The Seattle Heart Failure Model: prediction of survival in heart failure. Circulation. 2006; 113(11):1424-33.

14. Bernabeu-Wittel M, Moreno-Gaviño L, Ollero-Baturone M,Barón-Franco B, DíezManglano J, Rivas-Cobas C, et al., PROFUND researchers. Validation of PROFUND prognostic index over a four-year follow-up period. Eur J Intern Med. 2016;36:20-

15. Cid-Ruzafa J, Damián-Moreno J. Valoración de la discapacidad física: el indice de Barthel. Revista Española de Salud Pública. 1997; 71(2):127-137.

16. Rockwood K, Song X, MacKnight C, Bergman H, Hogan DB, McDowell I, et al. A global clinical measure of fitness and frailty in elderly people. CMAJ. 2005; 173(5): 489-495.

17. Ruiz-Laiglesia FJ, Sánchez-Marteles M, Pérez-Calvo JI, Formiga F, Bartolomé-Satué JA, Armengou-Arxé A, et al. Comorbidity in heart failure. Results of the Spanish RICA Registry. QJM: An International Journal of Medicine. 2014;107,12:989-994.

18. Pérez-Barquero M, Gutiérrez Román-Sánchez P. for Working Group on Heart Failure of the Spanish Society of Internal Medicine (SEMI-IC study). Comorbidity in patients hospitalized for heart failure in internal medicine departments. Rev Clin Esp. 2010; 210:149-58.

19. Health reform: Meeting the challenge of ageing and multiple morbidities, OECD Publishing (2011).

20. Rodríguez Pérez A, Alfaro Lara ER, Nieto Martín MD, et al. Deprescribing in patients with multimorbidity: a necessary process. Eur J Intern Med. 2015; 26:18-9 